\title{
Effecient Computation Methods for 3D Microscopic Ellipsometry
}

\section{Yia-Chung Chang ${ }^{1}$}

Research Center for Applied Sciences, Academia Sinica

128 Sec. 2, Academia Rd., Nankang, Taipei, Taiwan 11529

Email:yiachang@gate.sinica.edu.tw

Address, Country

\section{Huai-Yi Xie}

Research Center for Applied Sciences, Academia Sinica

128 Sec. 2, Academia Rd., Nankang, Taipei, Taiwan 11529

E-mail:damoxie@gate.sinica.edu.tw

We review theoretical models for describing light scattering from various distributions of micro/nano structures on a substrate. We review results obtained by rigorous coupled wave analysis (RCWA) and the multilayer Green's function (GF) method with geometry-adapted basis functions and compare the pros and cons. We show that the GF method is particularly useful for calculating the ellipsometry spectra of a variety of systems efficiently and accurately. The calculated ellipsometric spectra can be compared with experimental data for a large frequency range to extract the critical dimensions of structure features. Finally, we show how to use the GF method to compute signals of microscopic imaging ellipsometry efficiently, including examples on light scattering spectra for $\mathrm{ZnO}$ microspheres and the polarization-dependent reflectance images. 


\section{Introduction}

Spectroscopic ellipsometry(SE) is a well established technology for determining thin film thickness and its optical constants. In the last two decades, it has been used in semiconductor industry to inspect the critical-dimension (CD) of periodic patterns on semiconductor chips designed for integrated circuit.[1] Such a technology is often called "optical metrology". The critical dimension of features on a grating structure determined by optical metrology can achieve nanometer accuracy even though the wavelengths of light used are typically in the range of $200 \mathrm{~nm}-800 \mathrm{~nm}$. The method relies on accurate fitting of simulated results fof the ellipsometric parameters (i.e. ratios of reflectivities for s- and p- polarized light) to the corresponding experimental data for a presumed geometry described by a few adjustable parameters. Here the s- or p-polarized light refers to light with the electric or magnetic field perpendicular to the plane of incidence. Optical metrology is a noninvasive characterization method, which is capable of probing buried structures in a semi-transparent layer.

For optical metrology of the structure details of a design, an efficient alogrithm is needed in order to determine the critical-dimension in real-time. For one-dimensional (1D) periodic array of strcuture patterns on a multilayer substrate, the most popular computation method for optical metrology is the rigorous-coupled-wave analysis (RCWA). [2-10] In this method, the structure pattern is divided into many thin slices such that the dielectric-constant distribution in each slice can be considered $z$ independent, where $z$ is taken to be the axis perpendicular to the substrate. The electromagnetic fields in each slice are described as linear combinations of plane waves. A transfer matrix equation, which is obtained by matching boundary conidtions, is then solved to obtained the reflectivity. The computation time for such an algoritm scales like $\alpha M \cdot N^{3}$, where $N$ is the number of plane waves used and $M$ is number of slices needed to describe the variation of the structure pattern along the $z$ direction. $\alpha$ is a factor describing the payload of matrix diagonalization required in RCWA calculations.

For two-dimensional (2D) periodic arrays of identical strcuture patterns or isolated structures in on multilayer substrate, the RCWA approach can become rather inefficient if the optical contrast between the featured material and background is high. In this case, the number of plane waves $N$ needed to describe the electromagnetic field would be quite large. For example, a metallic structure embedded in a dielectric would require an expansion of the electro-magnetic fields in terms of least 50 planes in each direction, resulting an $N$ value $>2500$ for 2 D arrays. For such systems, numerical methods based on the multilayer Green's function (GF) approach become much more efficient. [11-14] The computation time for an algoritm based on the multilayer GF method scales like $v \cdot M \cdot N \cdot \ln N$ for periodic systems [11] and $v \cdot M \cdot N \cdot K$ for isolated structures, where $N$ is the number of plane waves needed in the expansion of the GF, $v$ is the number of iterations needed to solve the linear equation for light scattering, $M$ is the number of slices needed to describe the electromagnetic field inside the structure, and $K$ is the nmber of localized basis fuctions needed in each slice. For isolated structures with cylindrical or spherical symmetry, values of both $N$ and $K$ can be significantly reduced by using the symmetryadapted basis functions, making the method even more efficient. Thus the GF method 
with suitably chosen basis functions can be a very effective tool for calculating the ellipsometric spectra for light scattering from various nanostrues.

\section{Rigorous coupled-wave analysis}

The Maxwell's equations for a non-magnetic medium without source terms are given by:

$$
\begin{aligned}
& \nabla \cdot \mathbf{D}=0, \\
& \nabla \cdot \mathbf{B}=0, \\
& \nabla \times \mathbf{E}=-\frac{\partial \mathbf{B}}{\partial t}, \\
& \nabla \times \mathbf{H}=\frac{\partial \mathbf{D}}{\partial t},
\end{aligned}
$$

and

$$
\begin{aligned}
& \mathbf{D}=\varepsilon \mathbf{E} \\
& \mathbf{B}=\mu_{0} \mathbf{H} .
\end{aligned}
$$

Combining Eqs. (1) and (2) leads to the wave equation $\mu_{0}^{-1} \nabla \times \varepsilon^{-1} \nabla \times \mathbf{H}=-\frac{\partial^{2} \mathbf{H}}{\partial t^{2}}$ or $\nabla \times \varepsilon^{-1} \nabla \times \mathbf{H}=k_{0}^{2} \mathbf{H} \quad$ where $\quad \frac{\partial^{2} \mathbf{H}}{\partial t^{2}}=-\omega^{2} \mathbf{H} \quad$ and $\quad k_{0}=\frac{\omega}{c}$. Using coupled-wave expansions in the $x-y$ plane, we have $\mathbf{H}=H_{\ell}(z) e^{i\left(K_{x} x+K_{y} y\right)} \mathbf{e}_{\ell}$ where $H_{\ell}(z)$ is a column vector of dimension $N^{2}$ and $\ell\left(\mathbf{e}_{\ell}\right)$ represents the direction of magnetic field, $x$ for TE mode and $y$ for TM mode. $K_{x}, K_{y}$ denote $N^{2} \times N^{2}$ square matrices with diagonal matrix elements $\left(K_{x}\right)_{n n}=k_{0 x}+\frac{2 n \pi}{p_{x}} \quad$ and $\quad\left(K_{y}\right)_{m m}=k_{0 y}+\frac{2 m \pi}{p_{y}}$; $n, m=1,2, \ldots, N . k_{z}=-i \partial_{z}$ remains a scalar in our $N^{2}$-dimensional Hilbert space. Since $\nabla \cdot \mathbf{H}=0$, we have

$$
K_{z} H_{z}=-\left(K_{x} H_{x}+K_{y} H_{y}\right) \text {, }
$$

which then leads to the following linear equations for the magnetic fields[11]

$$
L(z)\left(\begin{array}{c}
H_{x} \\
H_{y}
\end{array}\right)=\left(\begin{array}{cc}
-\varepsilon k_{0}^{2}-K_{x}^{2}-\varepsilon K_{y} \varepsilon^{-1} K_{y} & -\varepsilon K_{y} \varepsilon^{-1} K_{x}-K_{x} K_{y} \\
-\varepsilon K_{x} \varepsilon^{-1} K_{y}-K_{y} K_{x} & -\varepsilon k_{0}^{2}-K_{y}^{2}-\varepsilon K_{x} \varepsilon^{-1} K_{x}
\end{array}\right)\left(\begin{array}{c}
H_{x} \\
H_{y}
\end{array}\right)=-k_{z}^{2}\left(\begin{array}{c}
H_{x} \\
H_{y}
\end{array}\right) .
$$

The relationship between the magnetic fields and the electric fields can be obtained from Maxwell's equations as

$$
\left\{\begin{array}{l}
-\varepsilon E_{x}=k_{z} H_{y}+K_{y}\left(K_{x} H_{x}+K_{y} H_{y}\right) k_{z}^{-1} \\
\varepsilon E_{y}=k_{z} H_{x}+K_{x}\left(K_{x} H_{x}+K_{y} H_{y}\right) k_{z}^{-1}
\end{array},\right.
$$

or 
$\left(\begin{array}{c}E_{y} \\ -E_{x}\end{array}\right)=\varepsilon^{-1} k_{z}\left(\begin{array}{l}H_{x} \\ H_{y}\end{array}\right)\left[1+\left(\begin{array}{cc}K_{x}^{2} & K_{x} K_{y} \\ K_{y} K_{x} & K_{y}^{2}\end{array}\right) k_{z}^{-2}\right]$.

Let $\Psi=\left(\begin{array}{c}H_{x} \\ H_{y}\end{array}\right)$ and $\Psi^{\prime}=\left(\begin{array}{c}E_{y} \\ -E_{x}\end{array}\right)$, we get the following differential equations

$\partial_{z}^{2} \Psi=L(z) \Psi \equiv \lambda \Psi$,

$\Psi^{\prime}=D(z) \partial_{z} \Psi$,

where

$D(z)=\varepsilon^{-1}\left[1-\left(\begin{array}{cc}K_{x}^{2} & K_{x} K_{y} \\ K_{y} K_{x} & K_{y}^{2}\end{array}\right) L(z)^{-1}\right]$.

The boundary conditions are: $\Psi$ and $\Psi^{\prime}$ continuous across a boundary along $z$. The matrix $L(z)$ can be diagonalized by a similarity transformation:

$S^{-1} L S=\Lambda=Q^{2}$,

where $\Lambda$ is the diagonal matrix formed by the eigenvalues $\left(\lambda_{i}\right)$ of $L, Q$ is a diagonal matrix with entries $q_{i}=\sqrt{\lambda_{i}}, S$ is the similarity transformation matrix whose columns are the eigenvectors of $L$. With the combination of decaying and growing waves, the wave function $\Psi(z)$ in each layer can be expressed as

$$
\begin{aligned}
& \Psi=\sum_{j} s_{j} e^{q_{j} z} f_{j}+\sum_{j} s_{j} e^{-q_{j} z} g_{j} \equiv S e^{Q z} f+S e^{-Q z} g, \\
& \partial_{z} \Psi \propto \sum_{j} s_{j} q_{j} e^{q_{j} z} f_{j}-\sum_{j} s_{j} q_{j} e^{-q_{j} z} g_{j} \equiv S Q e^{Q z} f-S Q e^{-Q z} g,
\end{aligned}
$$

where $f$ and $g$ are the vectors formed by $f_{i}$ 's and $g_{i}$ 's which are to be determined by the boundary conditions. The wave functions are arranged in such a way that $e^{q_{j} z}$ represents a forward propagating or decaying wave in the positive $z$-direction and is therefore the physical propagating wave in the substrate, while $e^{-q_{j} z}$ represents a backward propagating or growing wave and is therefore not allowed in the substrate.

Using the boundary conditions, we obtain the following transfer-matrix equation between any two consecutive layers $j$ and $j+1$

$$
\begin{aligned}
& \left(\begin{array}{l}
f_{j} \\
g_{j}
\end{array}\right)=\frac{1}{2}\left(\begin{array}{cc}
e^{Q_{j} d_{j}} & 0 \\
0 & e^{-Q_{j} d_{j}}
\end{array}\right)\left(\begin{array}{cc}
1 & 1 \\
1 & -1
\end{array}\right)\left(\begin{array}{cc}
M_{j} & 0 \\
0 & N_{j}
\end{array}\right)\left(\begin{array}{cc}
1 & 1 \\
1 & -1
\end{array}\right)\left(\begin{array}{c}
f_{j+1} \\
g_{j+1}
\end{array}\right) \\
& =\frac{1}{2}\left(\begin{array}{cc}
e^{Q_{j} d_{j}} & 0 \\
0 & e^{-Q_{j} d_{j}}
\end{array}\right)\left(\begin{array}{ll}
M_{j}+N_{j} & M_{j}-N_{j} \\
M_{j}-N_{j} & M_{j}+N_{j}
\end{array}\right)\left(\begin{array}{l}
f_{j+1} \\
g_{j+1}
\end{array}\right) \equiv T_{j}\left(\begin{array}{l}
f_{j+1} \\
g_{j+1}
\end{array}\right),
\end{aligned}
$$

where $M_{j} \equiv S_{j}^{-1} S_{j+1}$ and $N_{j} \equiv J_{j}^{-1} J_{j+1}$.

It is convenient to write

$$
\left(\begin{array}{c}
f_{j} \\
g_{j}
\end{array}\right)=\left(\begin{array}{c}
f_{j} \\
R_{j} f_{j}
\end{array}\right)
$$

Substituting Eq. (13) into Eq. (12) gives 


$$
\begin{aligned}
& f_{j}=e^{-Q_{j} d_{j}}\left(M_{j}^{+}+M_{j}^{-} R_{j+1}\right) f_{j+1} \\
& R_{j} f_{j}=e^{Q_{j} d_{j}}\left(M_{j}^{-}+M_{j}^{+} R_{j+1}\right) f_{j+1},
\end{aligned}
$$

which can be rewritten as

$$
\begin{aligned}
& f_{j+1}=\left(M_{j}^{+}+M_{j}^{-} R_{j+1}\right)^{-1} e^{Q_{j} d_{j}} f_{j} \equiv T_{j} e^{Q_{j} d_{j}} f_{j} \\
& R_{j}=e^{Q_{j} d_{j}}\left(M_{j}^{-}+M_{j}^{+} R_{j+1}\right)\left(M_{j}^{+}+M_{j}^{-} R_{j+1}\right)^{-1} e^{Q_{j} d_{j}}=e^{Q_{j} d_{j}}\left(M_{j}^{-}+M_{j}^{+} R_{j+1}\right) T_{j} e^{Q_{j} d_{j}},
\end{aligned}
$$

where $M_{j}^{ \pm}=\frac{1}{2}\left(M_{j} \pm N_{j}\right)$. Note that $R_{j}$ is a square matrix of dimension $2 N^{2}$, which can be called the "Reflection matrix". In a uniform medium (outside the grating layer), $R_{j}$ is diagonal with the upper (lower) half representaing reflection coefficients for $H_{x}\left(H_{y}\right)$ field. Here, $H_{y}$ is the TM component, while $H_{x}$ is related to the TE component ( $E_{y}$ field) via $E_{y}=-\frac{k_{0} H_{x}}{k_{0 \mathrm{z}}}$ in the uniform layer. So, $R_{\mathrm{TE}}=-R_{\mathrm{Hx}}$.

By taking advantage of the symmetry property of the shape of the objects in the periodic array, one can reduce the basis size by a factor proportional to the number of symmetry operations, which can speed up the computation significantly.

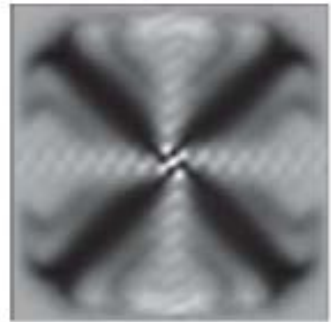

(a)

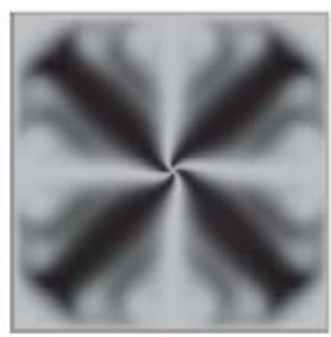

(d)

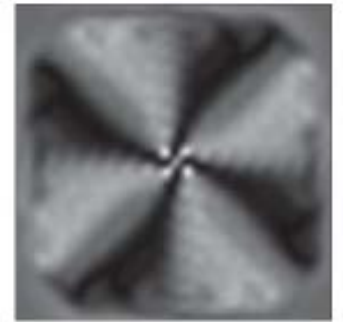

(b)

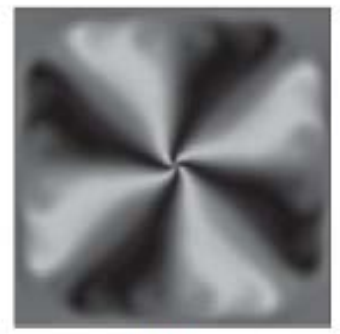

(e)

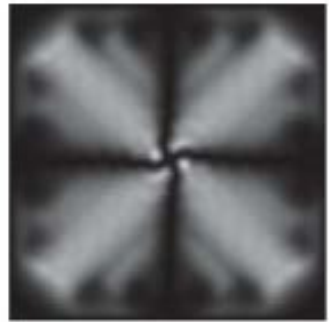

(c)

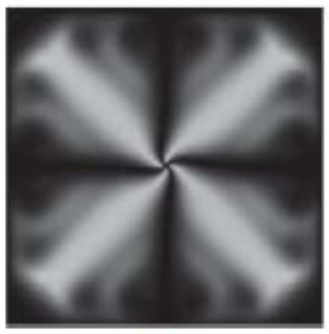

(f)

Fig. 1. Near-field transmittances of the VA cells in the emitted xy plane by the RCWA method, in which the absorption axes of the up (down) polarizers are set to be $\theta_{\text {up }}=\pi / 2\left(\theta_{\text {down }}=\pi / 2\right)$, and (a) $\varphi_{\text {up }}$ $=0\left(\varphi_{\text {down }}=0\right),(b) \varphi_{\text {up }}=\pi / 4\left(\varphi_{\text {down }}=0\right)$ and (c) $\varphi_{\text {up }}=\pi / 2\left(\varphi_{\text {down }}=0\right)$. Near-fields with corresponding set-ups by the Jones method are shown in (d), (e) and (f), respectively.

The RCWA method can also be applied for media consisting of periodic arrays of threedimensional birefringent (e.g. liquid-crystal) micro/nano structures. [15] The algorithm in Ref. 15 is formulated for stacks of micro/nano structures with isotropic or birefringent materials in an arbitrary order. Fig. 1 shows that the near-field transmittances are close to those obtained by the Jones method for small angles of 
dominant diffraction, while the far-field diffractions are quite different. [15] These results have been verified by using the finite-difference time-domain (FDTD) method. It is shown that the near-field transmittances approximate to those obtained by the Jones method as the angles of the dominant diffraction are small, while the far-field diffractions are verified by the finite-difference time-domain method, indicating the validity of the algorithm.

Recently, considerable efforts have been dedicated to the realization of 2D and 3D quasicrystal structures [16-18], in which the lack of translational symmetry is compensated by rotational symmetries not achievable by conventional periodic crystals, hence providing an alternative route for various optical applications in LEDs an dlasers [19]. By treating the quasicrystal structure as an irrational cut of a higher dimensional periodic structure, one can then apply the coupled-wave theory. Computation algorithms for simple $1 \mathrm{D}$ and $2 \mathrm{D}$ birefringent quasicrystals have been implemented to study the characteristic diffractions of the aperiodic structures.[20] Compared to traditional supercell method, the coupled-waves approach allows us to capture the entire infinite aperiodic quasicrystal in a single finite computational cell, albeit at only a finite resolution. Due to the large number of plane waves involved as we double the dimensionality (which changes the number of basis functions from $N$ to $M=N^{2}$ ). Fortunately, by using an importance sampling technique, one can reduce the number of basis functions $(M)$ needed to obtain convergent results back to around a few times of $N$.[20] The calculated results display proper convergence/accuracy with suitable truncation of Fourier components in an efficient way, and show good agreement with results obtained by the FDTD method. The diffraction/reflection behaviors of twodimensional (2D) quasicrystal surfaces under various polarization and angles of incidence are then studied. Comparison of our RCWA simulation results for a 2D octagonal quasicrystal layer with those obtained by FDTD is shown in Fig. 2.[20]
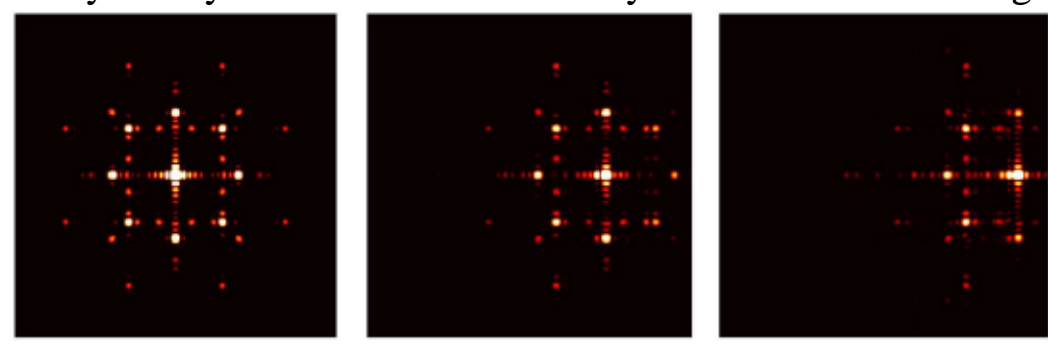

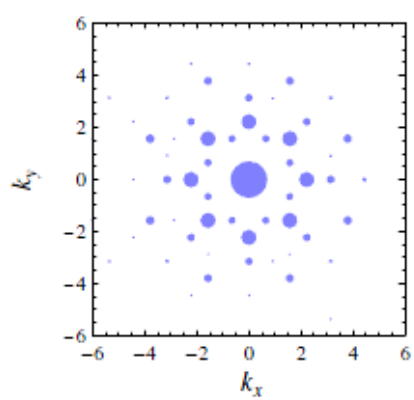

(a)

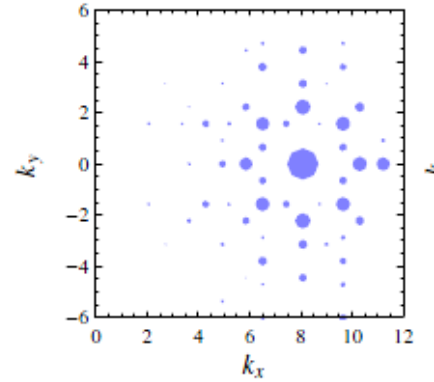

(b)

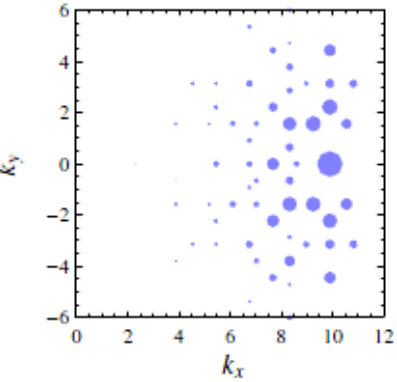

(c)

Fig. 2. Transmitted field $|\mathrm{E}|$ through a 2D octagonal quasicrystal layer calculated by FDTD (upper row) and RCWA (lower row) for three incident angles: (a) $\theta=0$, (b) $\theta=\pi / 4$, and (c) $\theta=\pi 3$. The thickness of the layer is $2 \mu \mathrm{m}$, and the number of Fourier components used is $\mathrm{Ng}=60$. 


\section{Multilayer Green's function approach}

Here we briefly review the GF method applied to a distribution of nanoparticles. Details of the method can be found in Refs. [11-14]. We write the wave function for the electric field in the form of linear combination of localized functions centered at sites where nanoparticles reside

$$
\mathbf{E}(\mathbf{r})=\frac{1}{\sqrt{N_{u}}} \sum_{i} e^{i \mathbf{k}_{0} \cdot \mathbf{R}_{i}} \mathbf{u}_{i}\left(\mathbf{r}_{i}\right),
$$

where $\mathbf{k}_{0}$ denotes the wave vector of the incident wave, $\mathbf{r}_{i} \equiv \mathbf{r}-\mathbf{R}_{i}$ denotes the spatial coordinate relative to a nanoparticle centered at $\mathbf{R}_{i}$ and $\mathbf{u}_{i}\left(\mathbf{r}_{i}\right)$ is a local function which is non-zero only for $\mathbf{r}$ in a cell surrounding $\mathbf{R}_{i} \cdot N_{u}$ is the number of nanoparticles in the sample area of interest. The Lipmann-Schwinger (L-S) equation for $N_{u}$ nanoparticles on a multilayer film reads,

$$
e^{i \mathbf{k}_{0} \cdot \mathbf{R}_{i}} \mathbf{u}_{i}\left(\mathbf{r}_{i}\right)=\sqrt{N_{u}} \mathbf{E}_{0}(\mathbf{r})+\sum_{j=1}^{N_{u}} \int d \mathbf{r}^{\prime} \mathbf{G}\left(\mathbf{r}, \mathbf{r}^{\prime}\right) \cdot V_{j}\left(\mathbf{r}^{\prime}\right) e^{i \mathbf{k}_{0} \cdot \mathbf{R}_{j}} \mathbf{u}_{j}\left(\mathbf{r}_{j}^{\prime}\right),
$$

where $\mathbf{r}$ is restricted in cell $i, \mathbf{G}\left(\mathbf{r}, \mathbf{r}^{\prime}\right)$ denotes the dyadic Green's function (GF) for the uniform multilayer background material, and $\mathbf{E}_{0}(\mathbf{r})$ denotes the unperturbed electric field (i.e. solution to the system without the nanoparticles). $V_{j}(\mathbf{r})=\left(\varepsilon_{a}-\varepsilon_{b}\right) k_{0}^{2} \theta\left(r<a_{j}\right)$ describes the perturbation due to replacing the dielectric constant of the background material (denoted by $\varepsilon_{b}$ ) by the one for nanoparticle (denoted by $\varepsilon_{a}$ ). $\theta\left(r<a_{j}\right.$ ) denotes a cut-off function, which is 1 for $\mathbf{r}$ inside the nanoparticle $j$ (with radius $a_{j}$ ) and 0 otherwise. We define the Fourier expansion of the GF suitable for a laminated structure stacked along the $z$ axis,

$\mathbf{G}\left(\mathbf{r}, \mathbf{r}^{\prime}\right)=\frac{1}{(2 \pi)^{2}} \int d \mathbf{k}_{n} e^{i \mathbf{k}_{n} \cdot\left(\boldsymbol{\rho}-\mathbf{\rho}^{\prime}\right)} \mathbf{g}_{n}\left(z, z^{\prime}\right)$,

where $\mathbf{g}_{n}\left(z, z^{\prime}\right)$ for a multilayer system is defined in Ref. [11]. Substituting Eq. (18) into Eq. (17) yields

$$
\mathbf{u}_{i}\left(\mathbf{r}_{i}\right)=\sqrt{N_{u}} e^{-i \mathbf{k}_{0} \cdot \mathbf{R}_{i}} \mathbf{E}_{0}(\mathbf{r})+\sum_{j=1}^{N_{u}} \int \frac{d \phi_{n}}{(2 \pi)^{2}} \int k_{n} d k_{n} \int d \mathbf{r}_{j}^{\prime} e^{i \mathbf{k}_{n} \cdot\left(\mathbf{p}_{i}-\mathbf{p}_{j}^{\prime}\right)} \mathbf{g}_{n}\left(z, z^{\prime}\right) \cdot e^{-i \mathbf{K}_{n} \cdot\left(\mathbf{R}_{j}-\mathbf{R}_{i}\right)} V_{j}\left(\mathbf{r}_{j}^{\prime}\right) \mathbf{u}_{j}\left(\mathbf{r}_{j}^{\prime}\right),
$$

where $\mathbf{K}_{n} \equiv \mathbf{k}_{n}-\mathbf{k}_{0}$. To solve Eq. (19), we expand the local function $\mathbf{u}_{i}\left(\mathbf{r}_{i}\right)$ in terms of some basis functions in the form

$$
\mathbf{u}_{i}(\mathbf{r})=\sum_{\mu} \mathbf{C}_{i, \mu} \Phi_{\mu}(\mathbf{r}) \text {, }
$$

where $\mathbf{C}_{i \mu}$ denote the vector expansion coefficients. Substituting (5) into (4) and projecting into the basis set leads to a linear equation

$$
\begin{aligned}
& \sum_{\mu} \mathbf{C}_{i \mu} \int d \mathbf{r}_{i} \Phi_{\mu^{\prime}}^{*}\left(\mathbf{r}_{i}\right) \Phi_{\mu}\left(\mathbf{r}_{i}\right)=\sqrt{N_{u}} e^{-i \mathbf{k}_{0} \cdot \mathbf{R}_{i}} \int d \mathbf{r}_{i} \Phi_{\mu^{\prime}}^{*}\left(\mathbf{r}_{i}\right) \mathbf{E}_{0}(\mathbf{r}) \\
& +\sum_{j=1}^{N_{u}} \int \frac{d \phi_{n}}{(2 \pi)^{2}} \int k_{n} d k_{n} \int d \mathbf{r}_{i} \Phi_{\mu^{\prime}}^{*}\left(\mathbf{r}_{i}\right) \int_{0}^{a_{j}} d \mathbf{r}_{j}^{\prime} e^{i \mathbf{k}_{n} \cdot\left(\mathbf{p}_{i}-\mathbf{p}_{j}^{\prime}\right)} \overrightarrow{\mathbf{g}}_{n}\left(z, z^{\prime}\right) \cdot e^{-i \mathbf{K}_{n} \cdot\left(\mathbf{R}_{j}-\mathbf{R}_{i}\right)} \sum_{\mu} \mathbf{C}_{i \mu} \Phi_{\mu}\left(\mathbf{r}_{j}^{\prime}\right) V_{j}\left(\mathbf{r}_{j}^{\prime}\right),
\end{aligned}
$$


which can be solved to obtain $\mathbf{C}_{i \mu}$ on all sites. Once the local functions at all sites are solved, the total field on a plane (taken at $z=0$ ) above the nanostructures can be obtained via

$$
\mathbf{E}(z=0)=\mathbf{E}_{0}(z=0)+\frac{1}{A_{\text {cell }}} \int d z^{\prime} \mathbf{g}_{0}\left(0, z^{\prime}\right) \cdot \int d \phi^{\prime} \int \rho^{\prime} d \rho^{\prime} e^{-i \mathbf{k}_{0} \cdot \boldsymbol{p}^{\prime}} \sum_{j} V_{j}\left(\mathbf{r}_{j}^{\prime}\right) \mathbf{u}_{j}\left(\mathbf{r}_{j}^{\prime}\right) .
$$

The TE (s-polarized) and TM (p-polarized) reflectivities are related to the total field at $z=0$ by $r_{s}=E_{y} / E_{o y}-1 ; \quad r_{p}=H_{y} / H_{o y}-1$, respectively, which can be used to obtain the ellipsometric parameters $\Psi=\tan ^{-1}\left|r_{p} / r_{s}\right| \square$ and $\Delta=\arg \left(r_{p} / r_{s}\right)$.

\subsection{Choosing the suitable basis functions}

If the nanoparticle has a spherical shape, the chosen symmetry-adapted basis functions are of the form

$$
\Phi_{\ell m}(\mathbf{r}) \equiv j_{\ell}\left(k_{1} r\right) Y_{\ell m}(\theta, \phi),
$$

where $j_{\ell}\left(k_{1} r\right)$ is the spherical Bessel function of order $\ell$ and $Y_{\ell m}(\theta, \phi)$ is the spherical harmonic function with quantum numbers $\ell, m$. If denote the cut-off value for $\ell$ as $\ell_{c}$, then the number of basis functions used is $K=\left(\ell_{c}+1\right)^{2}$. Note that all of these basis functions satisfy the Maxwell's equations for a uniform material with dielectric constant $\varepsilon_{a}$ (for the nanoparticle) if we choose $k_{1}^{2}=\varepsilon_{a} k_{0}^{2}$. The key advantage of using the spherical harmonics basis for solving EM fields is that for each angular function $Y_{\ell m}\left(\Omega^{\prime}\right)$ selected, the radial function $j_{\ell}\left(k_{1} r^{\prime}\right)$ is predetermined. Thus, the expansion of EM fields as described in (1) requires only a small set of unknown coefficients (determined by the maximum values of angular functions needed to obtain a convergent result), and the method becomes very efficient. For objects with diameter much smaller than the wavelength, the maximum value of $\ell$ needed can be as small as 2 (requiring only 9 basis functions). The method can be generalized to ellipsoidal objects via a coordinate scaling such that the objects become spherical in the new coordinate frame, and $\mathbf{u}_{i}(\mathbf{r})$ in each object is expanded in terms of spherical harmonics basis functions within its own scaled coordinates. For such method to work, one of the principal axes of the ellipsoid must be along $z$. In this proposal, we will further extend the method to objects of arbitrary shape via the use of hybrid basis functions. First, we fill the majority of the volume of the object with a minimum number of ellipsoids (with diameters larger than $5 \%$ of the wavelength). The remainder of the volume will be filled by small parallelipipes or tetrahedrons. (See Fig. 3). $\mathbf{u}_{i}(\mathbf{r})$ inside each

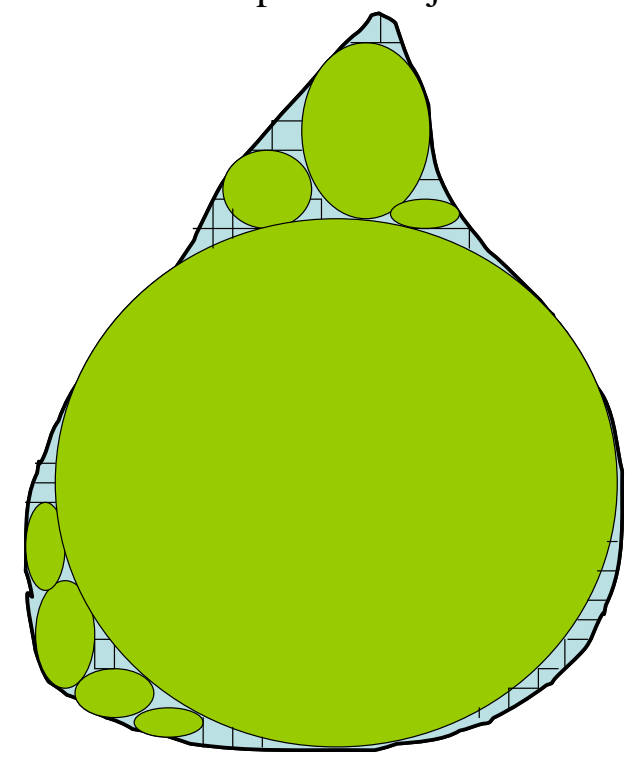
ellipsoid is expanded in terms of spherical harmonics basis functions defined in a scaled coordinate frame such

Fig. 3. Illustration of how to divide a general-shaped object into a few ellipsoids plus some finite elements. 
that the ellipsoid appears spherical. Outside the ellipsoids, $\mathbf{u}_{i}(\mathbf{r})$ is expanded in terms of finite-element basis functions for small parallelipipes or tetrahedrons. Since the majority of the object is filled by ellipsoids, we only need a small number of additional finite-elements to describe the solution. Thus, our hybrid basis functions (spherical harmonics in ellipsoids plus finite elements) serve as a good basis for finding the full solution efficiently.

\subsection{Periodic and random distribution}

The above description is valid for any distribution of a collection of nanoparticles. For periodic and random distributions of nanoparticles of identical shape and size, the problem can be further simplified. For periodic distributions, all local functions within the nanoparticle are identical. We have $\mathbf{u}_{i}\left(\mathbf{r}_{i}\right)=\mathbf{u}_{1}\left(\mathbf{r}_{i}\right)$ and $V_{j}\left(\mathbf{r}_{j}\right)=V_{1}\left(\mathbf{r}_{j}\right)$ at all sites. Thus Eq. (19) reduces to

$\mathbf{u}_{1}(\mathbf{r})=\sqrt{N_{u}} \mathbf{E}_{0}(\mathbf{r})+\sum_{n=1}^{N} \int d \mathbf{r}^{\prime} e^{i\left(\mathbf{k}_{0}+\mathbf{K}_{n}\right) \cdot\left(\mathbf{p}-\mathbf{\rho}^{\prime}\right)} \mathbf{g}_{n}\left(z, z^{\prime}\right) \cdot V_{1}\left(\mathbf{r}^{\prime}\right) \mathbf{u}_{1}\left(\mathbf{r}^{\prime}\right)$,

where $\mathbf{K}_{n}$ denote the reciprocal lattice vectors for the periodic array.

For random distributions, all local functions $\mathbf{u}_{i}\left(\mathbf{r}_{i}\right)$ may not be identical, but very similar. For a given site (say 1 ), we may approximate the local functions on other sites by $f \mathbf{u}_{1}\left(\mathbf{r}_{i}\right)$ on an average sense, where $\mathrm{f}$ is called a "similarity" factor, which should be between 0 and 1. [12] We shall consider this similarity factor as a fitting parameter. Then Eq. (20) reduces to

$\mathbf{u}_{1}(\mathbf{r})=\sqrt{N_{u}} \mathbf{E}_{0}(\mathbf{r})+\int \frac{d \phi_{n}}{(2 \pi)^{2}} \int k_{n} d k_{n} S\left(\mathbf{K}_{n}\right) \int d \mathbf{r}^{\prime} e^{i \mathbf{k}_{n} \cdot\left(\boldsymbol{\rho}-\boldsymbol{\rho}^{\prime}\right)} \mathbf{g}_{n}\left(z, z^{\prime}\right) \cdot V_{1}\left(\mathbf{r}^{\prime}\right) \mathbf{u}_{1}\left(\mathbf{r}^{\prime}\right)$,

where $\mathbf{K}_{n}=\mathbf{k}_{n}-\mathbf{k}_{0}$ and $S\left(\mathbf{K}_{n}\right)=1+f S_{1}\left(\mathbf{K}_{n}\right)$ is the structure factor with $S_{1}\left(\mathbf{K}_{n}\right)$ defined as

$S_{1}\left(\mathbf{K}_{n}\right)=\sum_{j \neq 1} e^{-i \mathbf{K}_{n} \cdot\left(\mathbf{R}_{j}-\mathbf{R}_{1}\right)} \mathrm{e}^{-\left(\mathbf{R}_{j}-\mathbf{R}_{1}\right)^{2} / 2 \lambda_{c}^{2}} \approx \frac{2 \pi \lambda_{c}^{2}}{A_{\text {cell }}} \mathrm{e}^{-K_{n}^{2} \lambda_{c}^{2} / 2}-\frac{2 \pi}{A_{\text {cell }}} \int_{0}^{R_{u}} J_{0}\left(K_{n} R\right) \mathrm{e}^{-R^{2} / 2 \lambda_{c}^{2}} R d R$,

where $\lambda_{\mathrm{c}}$ is the coherent length of light $[13,14]$ and $R_{u}=\sqrt{A_{\text {cell }} / \pi} . A_{\text {cell }}$ is the average area occupied by a nanoparticle on the sample surface.

\section{Simulation results for a few examples}

\subsection{Random distribution of Au nanoparticles}

In our previous works, we studied the ellipsometric spectra of a random distribution of Au nanoparticles [12] and effects of the presence of small clusters. [13,14] Scanning electron microscopy (SEM) pictures for the samples considered are shown in Fig. 4.(from [13]) Using Eq. (19) we can solve the local function $\mathbf{u}_{1}(\mathbf{r})$ inside a gold nanoparticle. Then, we use Eq. (22) to calculate the ellipsometric spectra for $\Psi$ and $\Delta$. We assume each nanoparticle has a spheroidal shape with lateral diameter $d$ and height 
$h$, and the nanoparticles are separated by an average distance $p$. We adjust the

(a)

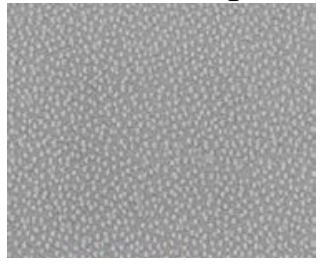

(b)

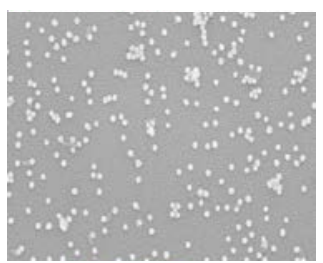

(c)

(d)

Fig. 4. SEM pictures of samples with random distribution of Au nanoparticles with diameters: (a) $20 \mathrm{~nm}$, (b) $40 \mathrm{~nm}$, (c) $60 \mathrm{~nm}$, and (d) $80 \mathrm{~nm}$.

parameters $d, h, p$, and $f$ for each sample until we get the best-fit for experimental spectra of $\Psi$ and $\Delta$ for three difference angles of incidence $\left(55^{\circ}, 60^{\circ}\right.$, and $\left.65^{\circ}\right)$. Another parameter, $\lambda_{c}$ (the coherent length of incident light) is chosen to be $3500 \mathrm{~nm}$, which gives the best over-all fit for all samples considered. The results are shown in Fig. 5, and the best-fit parameters along with the mean-square-error for each fit are listed in Table 1.

Here, we used cut-offs for basis functions and integration mesh points $\left(l_{\mathrm{c}}, N, M\right)=$ $(6,61,100)$ to ensure convergence,.[13] We find that the theoretical results agree reasonably well with SE measurements for the $20 \mathrm{~nm}$ case. For samples with larger size nanoparticles, the fit is not as good, especially for photon energies below $2.5 \mathrm{eV}$ as a result of the surface plasmonic effect.

Table 1. Best-fit parameters used in the theoretical modeling for Au nanoparticles without clusters.

\begin{tabular}{ccccc}
\hline $\begin{array}{c}\text { Particle } \\
\text { height } \\
h \\
(\mathrm{~nm})\end{array}$ & $\begin{array}{c}\text { Aspect } \\
\text { ratio } \\
(h / d)\end{array}$ & $\begin{array}{c}\text { Simila } \\
\text { rity } \\
\text { factor } \\
f\end{array}$ & $\begin{array}{c}\text { Averag } \\
\text { e pitch } \\
p(\mathrm{~nm})\end{array}$ & MSE \\
\hline 18 & 0.95 & 1.0 & 50 & 5.50 \\
\hline 38 & 0.90 & 0.8 & 140 & 8.00 \\
\hline 66 & 0.98 & 0.7 & 170 & 9.94 \\
\hline 84 & 0.97 & 0.7 & 220 & 11.46 \\
\hline
\end{tabular}



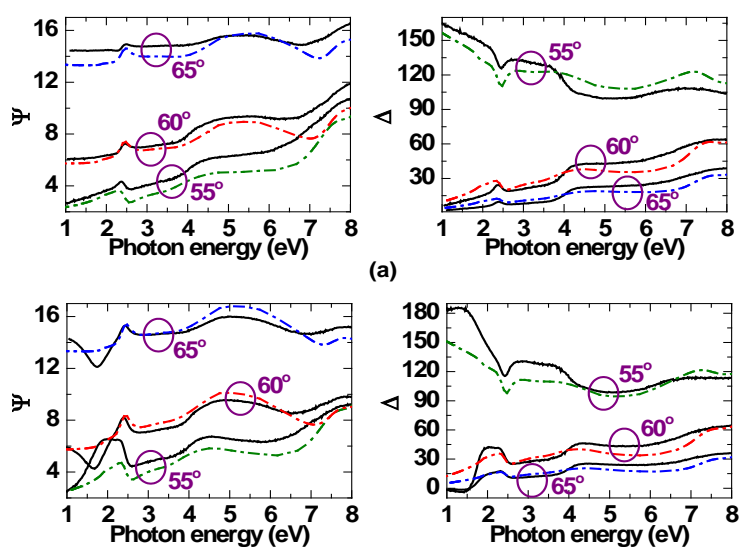

(b)
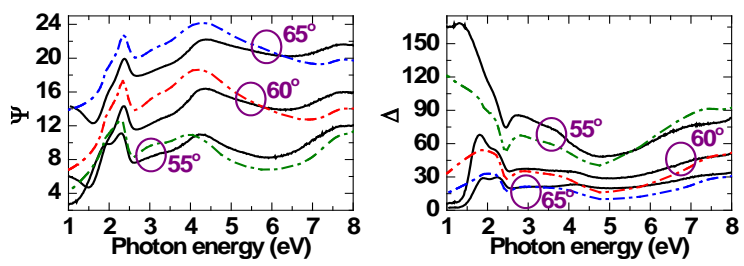

(c)
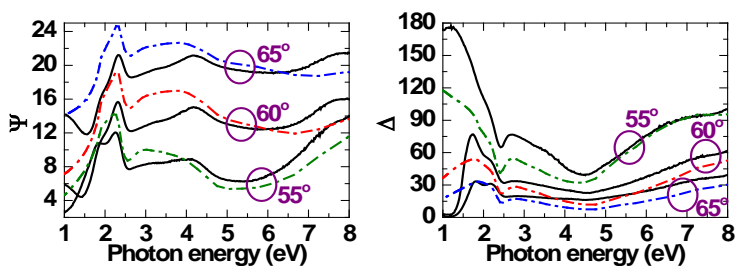

(d)

Fig. 5. SE measurements (solid curves) and model calculations (dash-dotted curves) without clusters for random distribution of nanoparticles with nominal sizes of (a) $20 \mathrm{~nm}$, (b) $40 \mathrm{~nm}$, (c) $60 \mathrm{~nm}$, and (d) $80 \mathrm{~nm}$ for incident angles of $55^{\circ}, 60^{\circ}$, and $65^{\circ}$.

\subsection{Clustering effect for random distribution of Au nanoparticles}

As shown in Fig. 4, there exist close-packed clusters of nanoparticles in samples with nominal values of d equal to $40 \mathrm{~nm}, 60 \mathrm{~nm}$, and $80 \mathrm{~nm}$. For simplicity, we can model these close-packed clusters of nanoparticles as pancakes with different diameters $d_{\alpha}$ (the equivalent spheroid model), which are embedded in a random distribution of nonaggregated nanoparticles and coupled to near-by nanoparticles. In order to describe such a complicated system, we use three types of local functions $\mathbf{u}_{1}(\mathbf{r})$ for non-aggregated nanoparticles which are randomly distributed nanoparticles with identical diameters, $\mathbf{c}_{\alpha}(\mathbf{r})$ for small clusters with types labeled by $\alpha$, and $\mathbf{p}(\mathbf{r})$ for large patch of clustering nanoparticles. $N_{u}, N_{c}$, and $N_{p}$ denotes the numbers of cells occupied by non-aggregated nanoparticles, clustering nanoparticles and large patch of clustering nanoparticles, respectively. The fractions of areas occupied by non-aggregated nanoparticles, clustering nanoparticles, and large patch of clustering nanoparticles, are denoted by $f_{u}=N_{u} / N_{t}, f_{c}=N_{c} / N_{t} \quad f_{p}=N_{p} / N_{t}$, repectively. Here $N_{t}=N_{u},+N_{c}+N_{p}$. We solve the local functions $\mathbf{u}_{1}(\mathbf{r})$ and $\mathbf{p}(\mathbf{r})$ by using Eq. (25) with proper values for $A_{\text {cell }}$. The local 
function $\mathbf{c}_{\alpha}(\mathbf{r})$ are obtained by solving Eq. (25), including the coupling to $\mathbf{u}_{1}(\mathbf{r})$. Namely,

$$
\mathbf{c}_{\alpha}(\mathbf{r})=\sqrt{N} \mathbf{E}_{0}(\mathbf{r})+\int \frac{d \phi_{n}}{(2 \pi)^{2}} \int k_{n} d k_{n} d \mathbf{r}^{\prime} e^{i \mathbf{k}_{n} \cdot\left(\boldsymbol{\rho}-\boldsymbol{\rho}^{\prime}\right)} \mathbf{g}_{n}\left(z, z^{\prime}\right) \cdot\left\{V_{\alpha}\left(\mathbf{r}^{\prime}\right) \mathbf{c}_{\alpha}\left(\mathbf{r}^{\prime}\right)+f_{u} f S_{1}\left(K_{n}\right) V_{1}\left(\mathbf{r}^{\prime}\right) \mathbf{u}_{1}\left(\mathbf{r}^{\prime}\right)\right\}
$$

Finally, we obtain the reflectivities by finding the electric field at $\mathrm{z}=0$ according to

$$
\mathbf{E}(z=0)=\mathbf{E}_{0}(z=0)+\frac{1}{A_{\text {cell }}} \int d z \mathbf{g}_{0}\left(0, z^{\prime}\right) \cdot \int d \phi^{\prime} \int \rho^{\prime} d \rho^{\prime} e^{-i \mathbf{k}_{0} \cdot \rho^{\prime}}\left[f_{u} V_{1}\left(\mathbf{r}^{\prime}\right) \mathbf{u}_{1}\left(\mathbf{r}^{\prime}\right)+\sum_{\alpha} p_{\alpha} V_{\alpha}\left(\mathbf{r}^{\prime}\right) \mathbf{c}_{\alpha}\left(\mathbf{r}^{\prime}\right)+f_{p} V_{1}\left(\mathbf{r}^{\prime}\right) \mathbf{p}\left(\mathbf{r}^{\prime}\right)\right] .
$$

Several pancakes with diameters evenly distributed between $2 d$ and $4 d$ are used in the modeling. Here we have assumed the fraction of cluster of type $\alpha$ is inversely proportional to the area of cluster $A_{\alpha}$. We then fit the experimental spectra for $\Psi$ and $\Delta$ again including the effects of clusters by adjusting the parameters $f_{c}$ and $f_{p}$. The cutoffs for basis functions and integration mesh points are $\left(l_{c}, N, M\right)=(6,61,100)$ to ensure convergence. The results are shown in Fig. 6, and the best-fit parameters along with the mean-square-error for each fit are listed in Table 2.[13]

Comparing the spectra in Figs. 5 and 6, we notice a qualitative difference in the spectral lineshape for photon energies below $2.5 \mathrm{eV}$ with and without the clustering effect. Including clustering effect, the $\Psi$ spectra display oscillatory behavior in the spectral features with a dip near $1.5 \mathrm{eV}$ and a broad peak covering $2 \mathrm{eV}$ to $2.5 \mathrm{eV}$, indicating effects due to coupling of multiple plasmonic resonances, which gives better agreement with the experimental data. Furthermore, for photon energies above $5 \mathrm{eV}$, the agreement with experimental data for the $\Psi$ spectra is improved considerably when we also mix in the contribution from the patches of closely packed nanoparticles. The over-all MSE also becomes small with the inclusion of clustering effect. It has been tested that the effect of clustering Au nanoparticles modeled by pan-cake like ellipsods is similar to the results obtained by using actual clusters of closely-spaced Au nanoparticles averaged over various sizes and orientations.[14]

Table 2. Best-fit parameters used in the theoretical modeling for Au nanoparticles with clusters.

\begin{tabular}{ccccc}
\hline $\begin{array}{c}\text { Particle } \\
\text { height } \\
h(\mathrm{~nm})\end{array}$ & $\begin{array}{c}\text { Aspect } \\
\text { ratio } \\
(h / d)\end{array}$ & $\begin{array}{c}\text { Fraction } \\
\text { of small } \\
\text { clusters, } f_{c}\end{array}$ & $\begin{array}{c}\text { Fraction of } \\
\text { nanoparticle } \\
\text { patches, } f_{p}\end{array}$ & MSE \\
\hline 38 & 0.90 & 0.015 & 0.005 & 5.60 \\
\hline 66 & 0.98 & 0.02 & 0.03 & 6.36 \\
\hline 84 & 0.97 & 0.025 & 0.03 & 6.55 \\
\hline
\end{tabular}



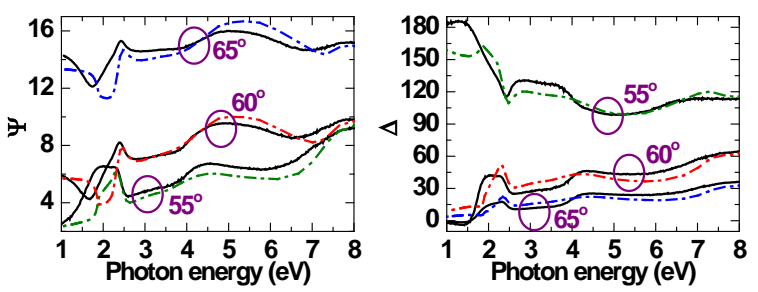

(a)
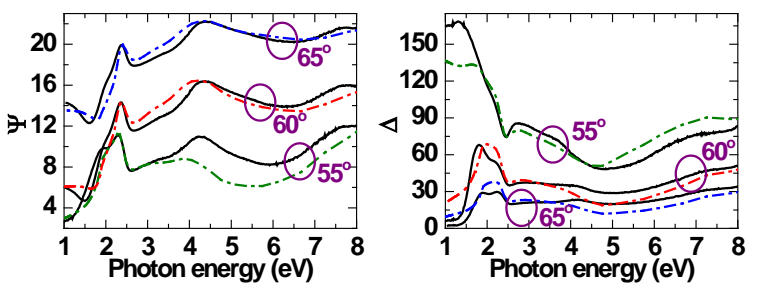

(b)
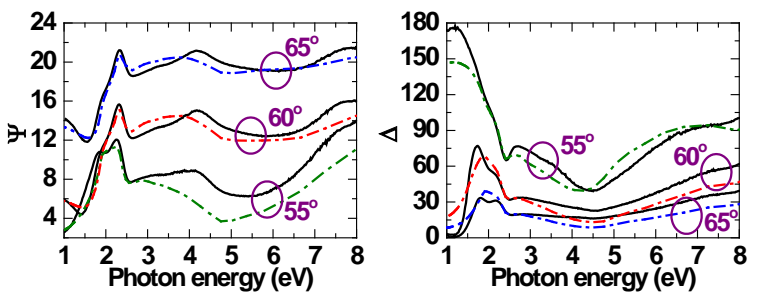

(c)

Fig. 6. SE measurements (solid curves) and model calculations (dashed dot curves) including clusters for random distribution of nanoparticles with nominal sizes of (a) 40, (b) 60, and (c) $80 \mathrm{~nm}$ for incident angles of $55^{\circ}, 60^{\circ}$, and $65^{\circ}$.

\subsection{Whisphering gallery modes of $\mathrm{ZnO}$ microspheres}

Recently, detailed studies on the optical cavity modes of zinc oxide microspheres under the optical excitation have been reported [21]. The zinc oxide microspheres with diameters ranging from 1.5 to $3.0 \mu \mathrm{m}$ were prepared using hydrothermal growth technique. The photoluminescence (PL) measurement of a single microsphere shows prominent resonances of whispering gallery modes at room temperature. The sharp resonances of whispering gallery modes in zinc oxide microspheres cover the entire visible window. They may be utilized in realizations of optical resonators, light emitting devices, and lasers for future chip integrations with micro/nano optoelectronic circuits, and developments of optical biosensors.

Figure 7 (taken from Ref. 21) shows the PL spectra of isolated $\mathrm{ZnO}$

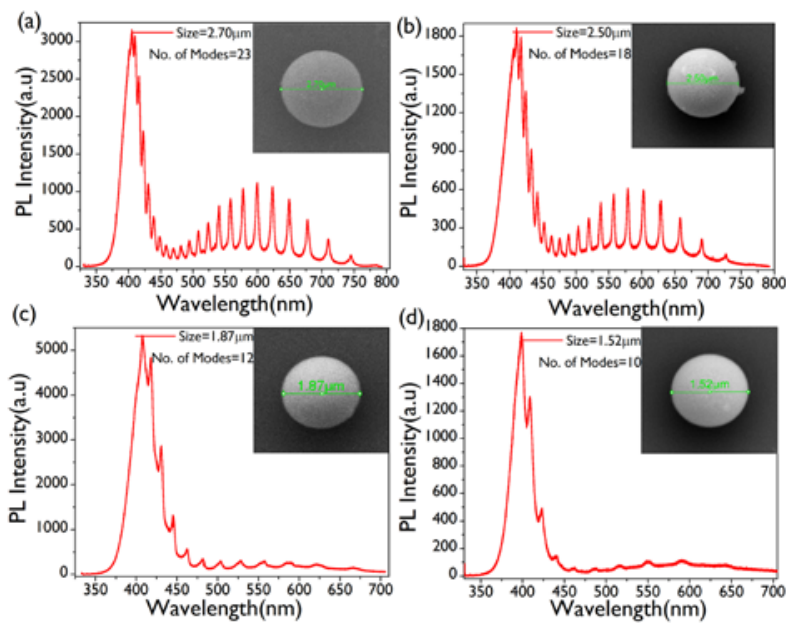

Fig. 7. PL spectra of a single $\mathrm{ZnO}$ microsphere placed on the Si substrate with diameter (a) $2.7 \mu \mathrm{m}$, (b) $2.5 \mu \mathrm{m}$, (c) $1.87 \mu \mathrm{m}$, and (d) $1.52 \mu \mathrm{m}$ 
microspheres with diameter (a) $D=2.70 \mu \mathrm{m}$, (b) $D=2.50 \mu \mathrm{m}$, (c) $D=1.87 \mu \mathrm{m}$, and (d) $D=1.52 \mu \mathrm{m}$. Many sharp peaks occur in the visible and near-IR range, which are attributed to the resonances associated with whispering gallery modes (WGMs). The experimentally observed whispering gallery modes in the photoluminescence spectrum can also be simulated by using the Spherical harmonics-based Green’s function (SHGF) method described above.

The simulation for the near-field intensity of light scattering from a $1 \mu \mathrm{m} \mathrm{ZnO}$ microsphere as a function wavelength is shown in Fig. 8(a). Also included for comparison is are results obtained by the Mie theory.[21] Excellent agreement between SHGF results and Mie Theory is obatined. Although the Mie theory is more accurate and efficient, it can only be applied to a free standing microscphere. The SHGF method can simulte a microsphere on a multilayer substrate, and examine the substrate effect, which can be significant on the near-field intensity observed on top of $\mathrm{ZnO}$ microsphere. The SHGF simulation results of the near-field for light scattering from a $1 \mu \mathrm{m} \mathrm{ZnO}$ microsphere placed on a $\mathrm{SiO}_{2}$ substarte coated with Au film of various thicknesses, $d$ are shown in Fig. 8(b). The near-field intensity for $\mathrm{ZnO}$ microsphere on the clean $\mathrm{SiO}_{2}$ substarte (without Au film) is shown by the black curve, which is much weaker than the free-standing microsphere shown in Fig. 8(a), since the contrast for $\mathrm{ZnO}$ on $\mathrm{SiO}_{2}$ is much smaller than that for $\mathrm{ZnO}$ on air. The near-field intensity is enhanced when the $\mathrm{Au}$ film is deposited, and the enhancement increases with the Au film thickness until the effect saturates at around $d=60 \mathrm{~nm}$. With the presence of Au film, the enhancement is largest near 550nm (where the plasmonic resonance occur) and the enhanced near field is stronger than the free-standing microsphere.
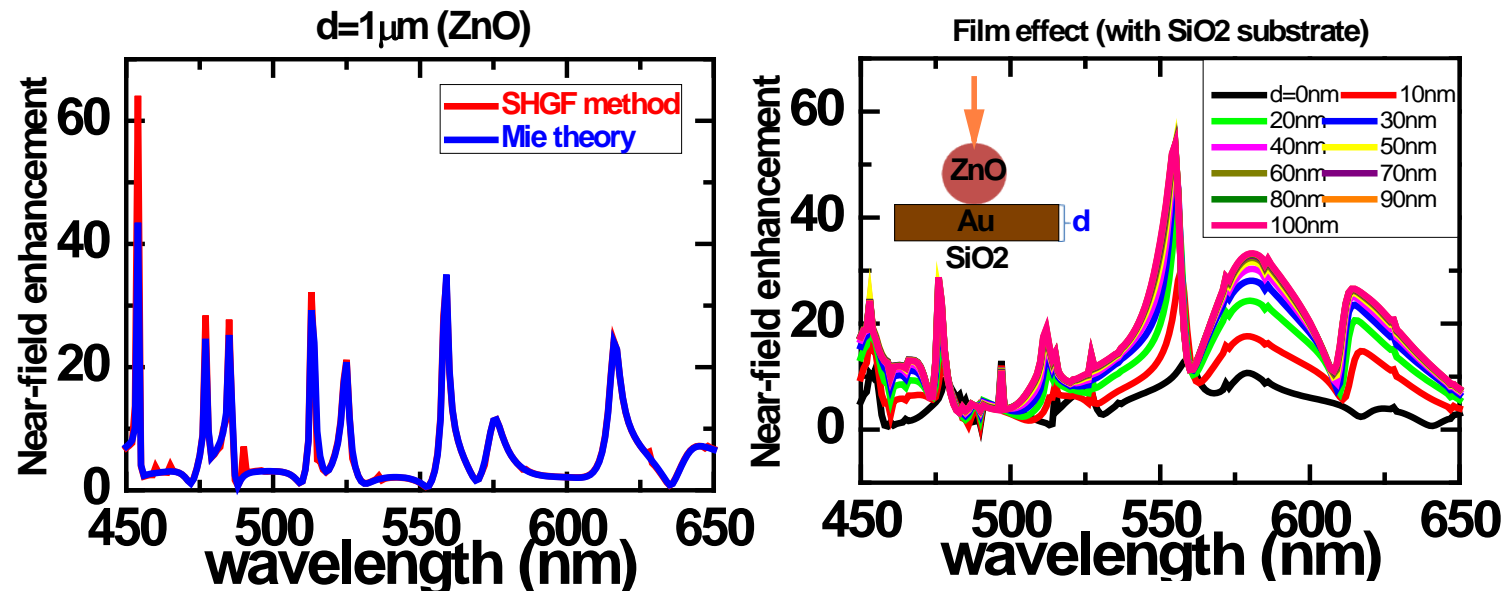

Fig. 8. Near-field for light scattering from a $1 \mu \mathrm{m} \mathrm{ZnO}$ microsphere as a function wavelength for (a) free standing case and (b) case with a $\mathrm{SiO}_{2}$ substrate coated with Au film of various thicknesses, $d$.

\subsection{Microscopic Imaging ellipsometry of $\mathrm{ZnO}$ microspheres}

In this section, we try to compute the microscopic imaging ellipsometry of an $\mathrm{ZnO}$ microsphere on the substrate (the material is glass). In Fig. 9, we present the calculated reflectance $R_{s}=\left|r_{s}\right|^{2}$ and $R_{p}=\left|r_{p}\right|^{2}$ for $s$ - and $p$-polarized where $r$ denotes the Fresnel 
reflection coefficient for an isolated $1 \mu \mathrm{m} \mathrm{ZnO}$ microsphere on $\mathrm{SiO}_{2}$ substrate with normal incidence at three different wavelengths: $450 \mathrm{~nm}, 550 \mathrm{~nm}, 650 \mathrm{~nm}$, respectively. The frame size $\mathrm{p}$ is chosen to be $3 \mu \mathrm{m}$. These images illustrae the interference pattern of polarization-resolved light reflection from a microsphere. For normal incidence, the images of $R_{s}$ and $R_{p}$ are related by a 90 -degree rotation. However, they will become different if detected by a microscopic imaging ellipsometer at a finite $\underset{\mathrm{Rp}}{\text { angle. }}$
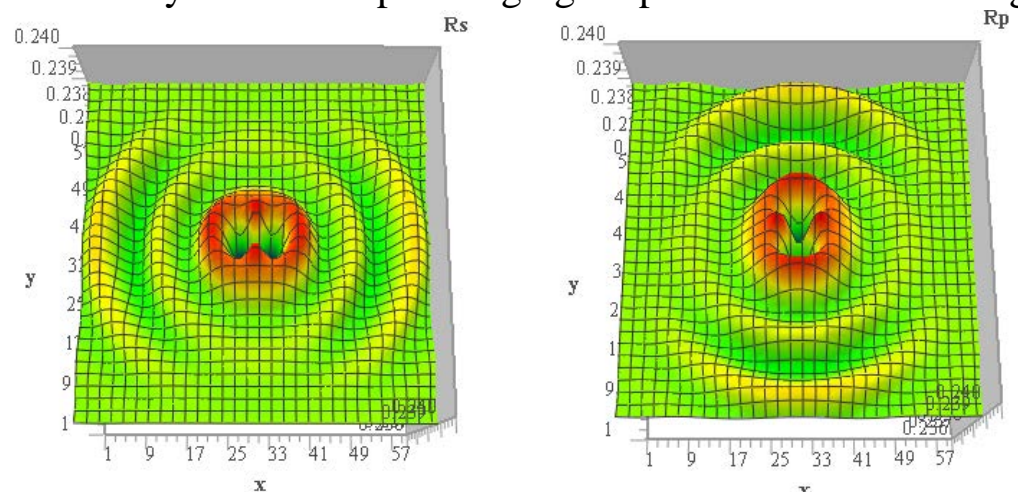

(a) Wavelength $=450 \mathrm{~nm}$
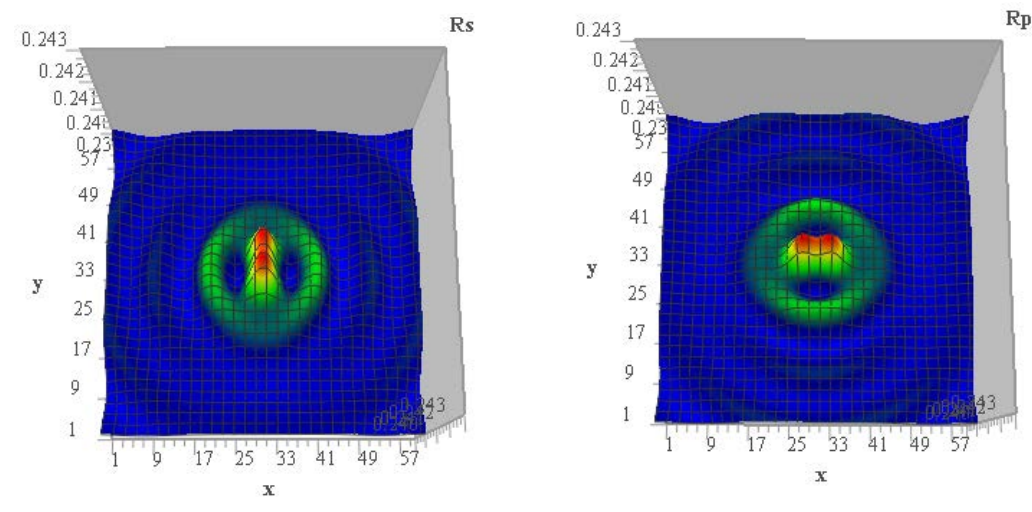

Rp

(b) Wavelength $=550 \mathrm{~nm}$
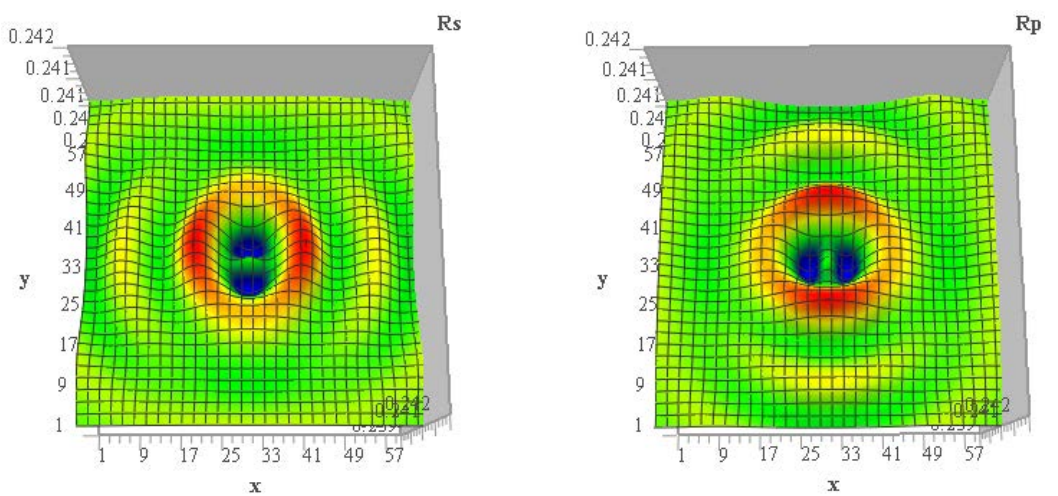

(c) Wavelength $=650 \mathrm{~nm}$

Fig. 9. Calculated reflectance $R_{s}$ and $R_{p}$ image for s- and p-polarized under three different wavelength $450 \mathrm{~nm}, 550 \mathrm{~nm}$ and $650 \mathrm{~nm}$, respectively. An normal incidence is used and the size of $\mathrm{ZnO}$ microsphere is $1 \mu \mathrm{m}$. The material of the substrate is $\mathrm{SiO}_{2}$. 


\section{Conclusion}

We have reviewed the RCWA and multilayer Green's function methods for studying various problems related to periodic/aperiodic distributions of nanostructures on or embedded in a multilayer film. With the use of spherical harmonics basis functions, the light scattering from a system involving ellipsoidal like objects can be handled efficiently and accurately. This technique allows us to analyze complicated arrangements of nanoparticles such as isolated nanoclusters or nanoclusters embedded in a random distribution of nanoparticles. Our modeling results for light scattering from such systems, including the coupling with clusters agree well with the experimental data obatined by spectroscopic ellipsometry. By adjusting the key parameters for describing the arrangements of nanoparticles of known properties in the fitting of ellipsometric spectra to the experiment, we can provide structural information of the distribution of nanoparticles, including the average size of nanoparticles, average spacing between adjacent particles, and the fraction of areas occupied by small clusters. Such information is very useful for nondestructive metrology of nanoparticles covered samples for application in nanofabrication. Furthermore, combining this optical metrology technique with an imaging ellipsometry, one can determine the 3D tomography of a semi-transparent structure and the distribution of its optical constant with micron-scale resolution laterally and nanoscale in depth, and at the same time the detailed arrangement of a few nanoscale objects inside the structure with nanoscale precision in 3D.

It has been shown that the RCWA method can be extended to liquid crystals (with birefringent media) and quasi-crystals (with quasi-periodic array) successively. For 2D quasi-crystals and $3 \mathrm{D}$ quasi-crystal slabs, it is still needs significant computation resource for RCWA calculation, since the computation time for RCWA would scale like $M^{3}$. In the GF method, we can expand the GF in terms of the same number of plane waves $(M)$ as adopted in RCWA (with importance sampling for $\mathbf{k}$ points in hyper-space). The computation time for the GF method would then scale like $M \times K \times v$, where $\mathrm{K}$ is the number of basis functions needed to describe the EM fields inside the objects in the unit cell and $v$ is the number of iterations needed to solve the linear equation (19). Since both $\mathrm{K}$ and $v$ are much smaller than $M$ for 2D or 3D quasi-crystals, the GF method with suitable local basis functions can become much more efficient. For future development, it would be desirable to augment the spherical harmonics functions (which are most suitable for ellipsoidal objects) with other local functions or finite elements in order to handle a more general class of objects.

\section{Acknowledgements}

This work was supported in part by Academia Sinica and National Science Council of the Republic of China under Contract No. NSC 101-2112-M-001-024-MY3.

\section{References}

[1] Fundamental solutions for real-time optical CD metrology, J. Opsal, H. Chu, Y. Wen, Y.C. Chang and G. Li, Proceedings of SPIE 2002, Metrology, Inspection and Process Control for Microlithography XVI, p. 163 (2002).

[2] M. G. Moharam, E. B. Grann, D. A. Pommet, and T. K. Gaylord, "Formulation for stable and efficient implementation of the rigorous coupled-wave analysis of binary gratings,” J. Opt. Soc. Am. A 12, 1068-1076 (1995). 
[3] M. G. Moharam, D. A. Pommet, E. B. Grann, and T. K. Gaylord, "Stable implementation of the rigorous coupledwave analysis of surface-relief gratings: enhanced transmittance matrix approach,” J. Opt. Soc. Am. A 12, 1077-1085 (1995).

[4] L. Li, "Formulation and comparison of two recursive matrix algorithms for modeling layered diffraction gratings,” J. Opt. Soc. Am. A 13, 1024-1035 (1996).

[5] L. Li, "Use of Fourier series in the analysis of discontinuous structures,” J. Opt. Soc. Am. A 13, 1870-1876 (1996).

[6] P. Lal'anne and G. M. Morris, "Highly improved convergence of the coupled wave method for TM polarization,” Opt. Soc. Am. A 13, 779-784 (1996).

[7] G. Granet and B. Guizal, "Really efficient implementation of the coupled-wave method for metallic lamellar gratings in TM polarization,” J. Opt. Soc. Am. A 13, 1019-1023 (1996).

[8] P. Lalanne, "Improved formulation of the coupled-wave method for two-dimensional gratings," J. Opt. Soc. Am. A 14, 1592-1598 (1997).

[9] G. Granet, "Reformulation of the lamellar grating problem hrough the concept of adaptive spatial resolution,” J. Opt. Soc. Am. A 16, 2510-2516 (1999).

[10] E. Popov and M. Neviére, "Grating theory: new equations in Fourier space leading to fast converging results for TM polarization,” J. Opt. Soc. Am. A 17, 1773-1784 (2000).

[11] Y. C. Chang, G. Li, H. Chu, and J. Opsal, "Efficient Finite-element Green's function approach for CD metrology of 3D gratings on multilayer films” J. Opt. Soc. Am. A 23, 638 (2006); US Patent US6867866B1.

[12] S.-H. Hsu, Y.-C. Chang, Y.-C. Chen, P.-K. Wei, Y. D. Kim, “Optical metrology of randomlydistributed Au colloids on a multilayer film”, Opt. Exp. 18, 1310-15 (2010); http://arxiv.org/abs/ 1305.6423.

[13] H.-Y. Xie, Y.-C. Chang, G. Li, S.-H. Hsu, "Effect of clustering on ellipsometric spectra of randomly distributed gold nanoparticles on a substrate”, Opt. Exp. 21, 3091-3102 (2013)

[14] H.-Y. Xie and Y.-C. Chang, "Light scattering from coupled plasmonic nanospheres on a substrate”, J. Opt. Soc. Am. B 30, 2215 (2013)

[15] I. L. Ho, Y. C. Chang, C. H. Huang, and W. Y. Li, “A detailed derivation of rigorous coupled wave algorithms for three dimensional periodic liquid-crystal microstructures, "Liquid Crystals, $\mathbf{3 8}$, 241-252 (2011).

[16] H. A. Bogart, and J. A. Rogers, "Three-dimensional nanostructures formed by single step, twophoton exposures through elastomeric penrose quasicrystal phase masks,” Nano Lett. 8, 2236-2244 (2008).

[17] G. Zito, B. Piccirillo, E. Santamato, A. Marino, V. Tkachenko, and G. Abbate, “Twodimensional photonic quasicrystals by single beam computer-generated holography,” Opt. Express 16, 5164-5170 (2008).

[18] M. Guo, Z. Xu, and X. Wang, "Photofabrication of twodimensional quasi-crystal patterns on UV-curable molecular Azo glass films,” Langmuir 24, 2740-2745 (2008).

[19]M. D. B. Charlton, M. E. Zoorob, and T. Lee, “Photonic quasicrystal LEDs: design, modelling, and optimisation”, Proc. SPIE 6486, 64860R (2007).

[20] I. L. Ho, M. T. Lee, Y.-C. Chang*, "Coupled-wave theory for birefringent photonic quasicrystal structures”, J. Opt. Soc. Am.B 29, 382-388 (2012).

[21] R. S. Moirangthem, P-J Cheng, P. C-H Chien, T. H. Ngo, S-W Chang, C-H Tien, and Y-C Chang, “Optical cavity modes of a single crystalline zinc oxide microsphere”, Optics Exp., 21, 30103020 (2013)

[22] G. Mie, Leipzig, “Beiträge zur optik trüber medien, speziell kolloidaler metallösungen,” Ann. Phys. 330, 377-445 (1908). 